\title{
Steps towards a System for Inferring the Interruptibility Status of Knowledge Workers

\author{
Lukas Ruge \\ lukas@lukas-ruge.info
} \\ Martin Christof Kindsmüller
mck@imis.uni-luebeck.de

\author{
Jörg Cassens \\ cassens@imis.uni-luebeck.de
}

\author{
Michael Herczeg \\ herczeg@imis.uni-luebeck.de
}

\author{
Institute for Multimedia and Interactive Systems (IMIS) \\ University of Luebeck \\ Ratzeburger Allee 160 \\ 23562 Luebeck, Germany \\ +49 451-500 5101
}

\begin{abstract}
In teams working closely together, interruptions of coworkers are normal and necessary. One of the goals of the ambient intelligent computing framework MATe (Mate for Awareness in Teams) is to prevent unwanted interruptions and at the same time improve social interaction. By creating awareness of each other's situation, users are able to judge how interruptible colleagues are. We describe the concept of MATe and its components and present related work on interruption handling and ontology-based reasoning as well as outline our current and future research in the area of context-aware systems.
\end{abstract}

\section{Categories and Subject Descriptors}

H.5 [Information Interfaces and Presentation]: Group and Organization Interfaces; I.4 [Artificial Intelligence]: Miscellaneous

\section{General Terms}

Design, Human Factors, Theory

\section{INTRODUCTION}

As the long foreseen era of disappearing computers begins and "technology recedes into the background of our lives" [23], our activities become input for ubiquitous, ambient intelligent applications that rely on surveillance and embedded systems for data acquisition. Such applications are able to appreciate the system's environment, be aware of persons in it, and respond intelligently to their needs [6]. Ambient intelligent applications may allow people to increase their awareness of one another at the work place and create a more effective and more pleasing work environment. The implementation of such services requires data to be collected on users and their environments from sensory devices. The sensory input captures specific aspects of the particular situation the user is in. In the next step, the ambient intelligent system tries to identify a more general context using the perceived data (context-awareness [14]). The deduced contextual information can then be distributed to services that can make further use of it (context-sensitivity [14]).

Availability of raw contextual data is no longer an issue. Coffee cups, keys, toys, refrigerators can be equipped with sensors and wireless connections and used to supply such data. Devices like smart phones, PDAs and notebooks, often capable of GPS localization, can be used to send and receive information at all times and places. Widespread use of microblogs, online calendar services and social networks supplies additional information on user activities and whereabouts. Thus, the question is no longer how to acquire data but rather what information is pertinent in ascertaining a specific user context as well as how to model the collected information and the context itself.

This paper is organized as follows. Section 2 analyzes related work on interruption, pervasive systems in work environments, context modeling and the search for emerging standards. Section 3 describes a framework of ambient intelligent applications for awareness among coworkers that is being implemented. The paper concludes in section 4 by laying out principles that guide the development of MATe's context model and tools.

\section{RELATED WORK}

According to Speier et al., interruptions are unexpected events that force people to move their focus away from their primary activity [18]. Dabbish and Baker focus on the importance of interruptions when assessing the interruptibility of a person [5]. Harr and Kaptelinin emphasize interactive aspects and establish that actors will usually look at priorities and available options [10]. Dabbish and Kraut found that displaying information which makes it possible to assess the interruptibility status of a person can lead to minimizing interruptions [4]. Tullio and others report on using displays at office doors to convey information about the occupant's interruptibility status [20].

Taking these findings from related work together it became obvious that we need complementary information from our prospective users to get a more complete picture. Therefore we decided to start our work on interruption management in the MATe framework with a survey to gain a better understanding for the particular problems, needs and expectations of the target users. Participants estimate that they spend between one and two hours a day on issues not planned beforehand due to unexpected interaction with other people. The majority of participants believe that informing potential visitors about the activity of the occupant of an office would prevent unnecessary interruptions while leaving it to 
the visitor to judge the importance of the interaction. Some presume that it would suffice to ask potential visitors not to disturb without revealing the occupant's activity.

After evaluating 602 hours of video material, Hudson, Fogarty et al. [9] [11] determine that only a small number of sensors are needed to determine the interruptibility of knowledge workers. Using a statistical model and the Subtle toolkit [8], they created a personalized statistical model that used sensory input to learn situations. The statistical model was implemented with decision trees, naïve Bayes predictors, support vector machines and decision stumps, yielding similar results with all techniques. The results reveal that as little as five information sources can be sufficient to provide a model with enough information to predict the interruptibility of participants with about $80 \%$ accuracy: a microphone to detect conversations, a sensor determining whether the phone is in use, information on the activity of mouse and keyboard as well as the time of the day.

Knox et al. [13], in contrast, rely on as much information as they can get, on the "cloud of devices", for rich and detailed information for their Scatterbox system. The Scatterbox is a "moving letterbox" that delivers messages to different devices based on the user's location and interruptibility. The context is modeled in the Web Ontology Language (OWL) ${ }^{1}$ and is corresponding to a mathematical description.

In Scatterbox, context is a tuple of subject, predicate and object. The subject is an entity of an OWL class like a person or place that has properties connecting it to other entities like a person's name or phone number. The situation a person is in is described as a set of such context tuples.

Another model for ambient intelligent applications is the collection of OWL ontologies ONTONYM [19]. ONTONYM also makes use of the idea of entities with certain properties to construct the context.

Wang et al. propose the CONON OWL ontology [22]. Specifically designed for logic reasoning, the idea is to reason about highlevel context information like activity or availability from lowlevel contextual information. The ontology has several classes like person, location, activity, and device. Each of them is associated with a wide range of object properties like name, age, temperature. As in Knox et al. [13], the low-level contextual data is represented using tuples of subject, object and predicate. CONON allows for reasoning based on the ontology itself as well as based on user defined rules.

OWL has been utilized to represent context in further ontologies like SOUPA [3]. SOUPA is used in the agent based architecture for ambient intelligent systems CoBrA [2].

Independent of the representation of context, Murdoch and Nixon [15] propose a framework that unifies existing methodologies for situation identification such as case-based reasoning, support vector machines, neural networks, decision trees, hidden markov models and bayesian networks.

Several projects examine the idea of creating an encapsulating structure like a middleware to identify situations in their knowledge representation. CAMUS is a "comprehensive middleware solution” [16], which supports different reasoning techniques to be used on an OWL Ontology. Other research has focused on

\footnotetext{
${ }^{1}$ http://www.w3.org/TR/owl-features/
}

architectural issues as well. Zhang and others present an infrastructure for managing and developing context-aware applications for smart homes unifying service-oriented with feature- and device-oriented approaches [24]. Voida et al. present a system that integrates information from the digital and the physical realm to help knowledge workers deal with large amounts of information [21]. Ejigu and others use OWL to support a pervasive system supporting collaboration of students and staff [7].

\section{MATE FRAMEWORK}

Within the MATe framework we are currently developing a series of prototypes addressing the need for interruption management identified in the literature and supported by the survey mentioned. MATe is an ambient intelligent, context-aware system that aims at improving situation awareness in work teams [17]. It is a clientserver-based collection of systems that are designed to improve the communication flow in the research group. The framework caters for different kinds of knowledge representations and reasoning mechanisms. The first implementation uses a logic-based reasoner and OWL, methodologies that have shown their benefits in the existing body of research.

The central component of MATe is the Awareness Hub. The hub is connected to all other applications and devices via the Extensible Messaging and Presence Protocol (XMPP) ${ }^{2}$. It handles the communication among the components and takes care of the correct routing of messages.

Within MATe a range of applications is connected to this Hub to sense and display contextual information. A central goal is to develop methods for better handling of interruptions of coworkers. An electronic, interactive door plate is one of the means to this end. A small touch screen display is mounted at every office door, showing the name of the occupant, his interruptibility status and, if so inclined, further information. This could be her or his current activity or location, if not being present in the office. The door plate enables visitors to assess the interruptibility status of the occupant and weigh it against the importance of their own intentions.

The input information for MATE comes from several sources, which will be extended by additional sensors over time, if necessary. A so-called drop zone is a specific area on every user's desk, which is able to detect unique tokens that are assigned to MATe users. It thus indicates the user's presence in the room. A desktop application lets users enter information directly into the system. Short messages can be sent and received via mobile phones through a gateway that is part of MATe.

In the future, social network feeds as well as users' calendars can be read, providing additional input. While the hub receives information from several sensors and services, it is not capable of reliably determining a user's position, activity or interruptibility, which would be crucial to implement the envisioned services. The hub focuses on sensor integration (perception) and does not realize further steps, which are to identify the context from input data (awareness) and to generate output or act on such identified contexts (sensitivity). Therefore, it is necessary to design a knowledge representation and implement reasoning capabilities, which can supply the hub (and through it the applications) with discerned high-level context information like activity, location or interruptibility.

\footnotetext{
${ }^{2}$ http://www.xmpp.org/
} 


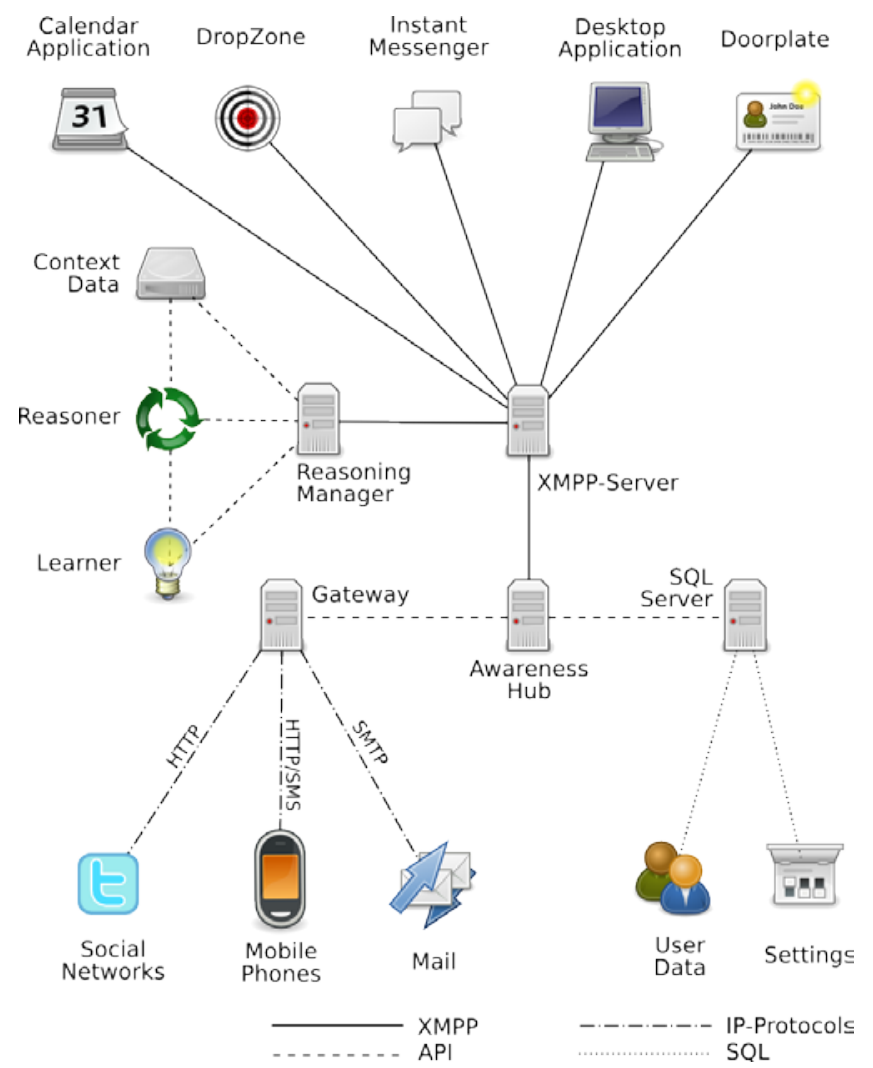

Figure 1: MATe Architectural Overview

\section{CONCLUSIONS}

While OWL seems to be established as a well defined data structure to be used in the modeling of context, the details on how to create ontologies remain discussed in the literature. Several aspects have to be considered as we create the ontology for use in MATe:

1. The model should be in line with emerging standards.

2. Depending on to which extent the work place allows for individualization of practices, the context model will have to be adjustable for differences in work flow.

3. Which data is useful to determine the necessary contextual information depends on contextual and personal aspects as well as on the application. Therefore, the model should allow for adaptation and should not define certain attributes to be relevant or irrelevant.

4. To protect the privacy of users, the model should require as little information as possible. Information needed by the reasoning engine should not necessarily be visible outside, for example to coworkers.

5. To cater for as many services as needed, the model should be able to incorporate any information given.

6. The systems should not just model the information itself but be able to model the certainty and accuracy of this information.

7. The model should cater for different types of contextual information and not only interruptions. This helps adaptation for the other envisioned usage scenarios.

We have developed an ontology based on OWL to be used to store a context model for MATe that will fulfill these requirements. In MATe's first incarnation, a reasoner based on descrip- tion logics is used to infer context information from the input data provided.

\section{OUTLOOK}

Making use of a middleware that encapsulates the details of the representation and the reasoning processes from the overall framework has emerged as a standard approach. A tool that coordinates the reasoning as well as updates values of entities in the knowledge base is part of MATe. The tool is able to provide the calculated high-level context information like interruptibility to the awareness hub. In order to provide for differences in users or changes over time, it is planned to incorporate machine learning modules that may be used to improve the ontology based on previously collected data and feedback from the user. All contextawareness tools will be connected to the hub via a reasoning manager using an open protocol.

Interruptibility of knowledge workers is just one of the issues we want to address with MATe. Assessing a user's activity to deliver information pro-actively is another area we are looking into. Context-dependent routing of messages (email, SMS, instant messages) is already realized rudimentary within MATe and could be enhanced by taking more factors into account. While the support of teams of knowledge workers is the principal target, the methods developed and framework implemented are applicable in other domains as well.

Since the discussion on how to best determine high-level context information is ongoing and no reasoning technique has produced clearly superior results yet, we plan to utilize different reasoning systems working on the contextual information provided by MATe's applications. We envision a blackboard architecture [12] to share the sensor data within MATe as well as to share results of individual reasoners between all reasoning systems. This architecture allows for competition and collaboration of reasoning technologies and for the use of meta-reasoning systems that may be used in the future to increase the accuracy of MATe's predictions.

\section{REFERENCES}

[1] Cassens, J., Kofod-Petersen, A., Zacarias, M., and Wegener, R., editors 2010. Proceedings of the Sixth International Workshop on Modelling and Reasoning in Context (Lisbon, Portugal, August 2010). CEUR Workshop Proceedings, CEUR-WS.org.

[2] Chen, H. 2004. An Intelligent Broker Architecture for Pervasive Context-Aware Systems. PhD thesis, University of Maryland, Baltimore County, December 2004.

[3] Chen, H., Finin, T., and Joshi, A. 2005. The soupa ontology for pervasive computing. In Calisti, M., Walliser, M., Brantschen, S., Herbstritt, M., Tamma, V., Cranefield, S., Finin, T., and Willmott, S., editors. Ontologies for Agents: Theory and Experiences. Birkhäuser, Basel. 233-258.

[4] Dabbish, L. A. and Kraut, R. E. 2004. Controlling interruptions: Awareness displays and social motivation for coordination. In Herbsleb, J. and Olson, G., editors. Proceedings of the 2004 ACM conference on Computer supported cooperative work. ACM, New York, NY, USA. 182-191

[5] Dabbish, L. A. and Baker, R. S. 2003. Administrative assistants as interruption mediators. In Conckton, G. and Korhonen, P., editors. CHI '03 extended abstracts on Human factors in computing systems. ACM, New York, NY, USA, 1020-1021. 
[6] Ducatel, K., Bogdanowicz, M., Scapolo, F., Leijten, J., and Burgelman, J.-C. 2001. ISTAG scenarios for ambient intelligence in 2010. Technical report. IST Advisory Group.

[7] Ejigu, D., Scuturici, M., and Brunie, L. 2008. Hybrid Approach to Collaborative Context-Aware Service Platform for Pervasive Computing. Journal of computers, 3(1):40.

[8] Fogarty, J. and Hudson, S. E. 2007. Toolkit support for developing and deploying sensor-based statistical models of human situations. In CHI '07: Proceedings of the SIGCHI conference on Human factors in computing systems. ACM, New York, NY, USA, 135-144.

[9] Fogarty, J., Hudson, S. E., Atkeson, C. G., Avrahami, D., Forlizzi, J., Kiesler, S., Lee, J. C., and Yang, J. 2005. Predicting human interruptibility with sensors. ACM Trans. Comput.-Hum. Interact., 12(1):119-146.

[10] Harr, R. and Kaptelinin, V. 2007. Unpacking the social dimension of external interruptions. In Field, M. and Lohr, K., editors. GROUP '07: Proceedings of the 2007 international ACM conference on supporting group work. ACM, New York, NY, USA, 399-408.

[11] Hudson, S. E., Fogarty, J., Atkeson, C. G., Avrahami, D., Forlizzi, J., Kiesler, S., Lee, J. C., and Yang, J. 2003. Predicting human interruptibility with sensors: A wizard of oz feasibility study. In Cockton, G. and Korhonen, P., editors. CHI '03: Proceedings of the SIGCHI conference on Human factors in computing systems. ACM, New York, NY, USA, 257-264.

[12] Jagannathan, V., Dodhiawala, R., and Baum, L. S., editors 1989. Blackboard Architectures and Applications. Academic Press, Inc., Orlando, FL, USA.

[13] Knox, S., Shannon, R., Coyle, L., Clear, A. K., Dobson, S., Quigley, A. J., and Nixon, P. 2008. Scatterbox: Contextaware message management. Revue d'Intelligence Artificielle, 22(5):549-568.

[14] Kofod-Petersen, A. and Aamodt, A. 2006. Contextualised ambient intelligence through case-based reasoning. In RothBerghofer, T. R., Göker, M. H., and Güvenir, H. A., editors. Proceedings of the Eighth European Conference on CaseBased Reasoning (ECCBR 2006). Volume 4106 of Lecture Notes in Computer Science. Springer, Berlin/Heidelberg, 211-225

[15] Murdoch, O. and Nixon, P. 2010. A unifying framework for situation identification methodologies. In Cassens et al. [1]. 49-60.
[16] Ngo, H. Q., Shehzad, A., Liaquat, S., Riaz, M., and Lee, S. 2004. Developing context-aware ubiquitous computing systems with a unified middleware framework. In Yang, L. T., Guo, M., Gao, G. R., and Jha, N. K., editors. Embedded and Ubiquitous Computing. Volume 3207 of Lecture Notes in Computer Science. Springer Berlin/Heidelberg. 239-247.

[17] Schmitt, F., Cassens, J., Kindsmüller, M. C., and Herczeg, M. 2010. Mental models of disappearing systems: Challenges for a better understanding. In Cassens et al. [1]. 6172.

[18] Speier, C., Valacich, J. S., and Vessey, I. 2003. The Effects of task interruption and information. In Janice, K. K. and DeGross, I., editors. Proceedings of the Eighteenth International Conference on Information Systems, 21-36.

[19] Stevenson, G., Knox, S., Dobson, S., and Nixon, P. 2009. Ontonym: a collection of upper ontologies for developing pervasive systems. In Gomez-Perez, J., editor. CIAO '09: Proceedings of the $1^{\text {st }}$ Workshop on Context, Information and Ontologies, 1-8.

[20] Tullio, J., Dey, A. K., Chalecki, J., and Fogarty, J. 2007. How it works: A field study of non-technical users interacting with an intelligent system. In Rosson, M. B. and Gilmore, D. J., editors. CHI '07: Proceedings of the SIGCHI conference on Human factors in computing systems. ACM, New York, NY, USA, 31-40.

[21] Voida, S., Mynatt, E., Macintyre, B., and Corso, G. M. 2002. Integrating virtual and physical context to support knowledge workers. IEEE Pervasive Computing, 1:73-79.

[22] Wang, X. H., Gu, T., Zhang, D. Q., and Pung, H. K. 2004. Ontology based context modeling and reasoning using OWL. In $2^{\text {nd }}$ IEEE Conference on Pervasive Computing and Communications Workshops (PerCom '04). IEEE Computer Society, 18-22.

[23] Weiser, M. 1991. The computer for the $21^{\text {st }}$ century. Scientific American. (September 1991), 94-104.

[24] Zhang, D., Wang, X., Leman, K., and Huang, W. 2003. OSGi based service infrastructure for context aware connected homes. In Mokthari, M., editor. Independent living for persons with disabilities and elderly people: Proceedings of the $1^{\text {st }}$ International Conference on Smart Homes and Health Telematics. IOS Press, 81-88. 